\title{
Space and Time in the Foundations of Mathematics, or some challenges in the interactions with other sciences ${ }^{1}$
}

\author{
Giuseppe Longo \\ Département d'Informatique \\ CNRS - Ecole Normale Supérieure, \\ 45, Rue d'Ulm, 75005 Paris (Fr.) \\ +3314432-3328 (fax -2151) longo@di.ens.fr \\ http://www.di.ens.fr/users/longo
}

Summary: Our relation to phenomenal space has been largely disregarded, and with good motivations, in the prevailing foundational analysis of Mathematics. The collapse of Euclidean certitudes, more than a century ago, excluded "geometric judgments" from certainty and contributed, by this, to isolate the foundation of Mathematics from other disciplines. After the success of the logical approach, it is time to broaden our foundational tools and reconstruct, also in that respect, the interactions with other sciences. The way space (and time) organize knowledge is a cross-disciplinary issue that will be briefly examined in Mathematical Physics, Computer Science and Biology. This programmatic paper focuses on an epistemological approach to foundations, at the core of which is the analysis of the "knowledge process", as a constitutive path from cognitive experiences to mathematical concepts and structures.

\section{Contents:}

1. The geometric intelligibility of space

1.1-2-3 Euclid, B. Riemann, A. Connes

1.4 Some epistemological remarks on the Geometry of Physical Space

2. Codings

2.1 Geometry in Computing

3. Living in space and time (towards Biology and Cognition)

3.1 Multiscale phenomena and the mathematical complexity of the neural system

4. Theories Vs Models

5. Conclusion: epistemological and mathematical projects

5.1 Epistemology

5.2 Geometry in Information

5.3 Geometric Forms and Meaning

\footnotetext{
${ }^{1}$ Invited Lecture, first American Mathematical Society/SMF Conference, Lyon, July, 2001.
} 


\section{The geometric intelligibility of space}

«The primary evidence should not be interchanged with the evidence of the "axioms"; since the axioms are mostly the result already of an original formation of meaning and they already have this formation itself always behind them» [Husserl, The origin of Geometry, 1933].

Man has always been organizing and giving meaning to space. This was done by action, gestures and language; Mathematics, Geometry in particular, provided the most stable conceptual reconstructions of phenomenal space. I will try to find a methodological unity to its highest moments, when geometric tools unified, and still now unify, the space of senses and physical space or different forms of mathematical understanding of space. To this aim, and by a rather arbitrary choice, I will stress the unity in the questioning by Euclid, Riemann and Alain Connes²: the issue here is not the 'names' of the mathematicians mentioned, nor their individual contribution, which may interest the historian, but the focus on mathematical theories which may soundly refer to them.

The claim is that space, in these three paradigmatic approaches, is made intelligible by proposing different answers to similar "questions": How do we access to space? How do we measure it? By which operators do we act on it?

\subsection{Euclid}

Euclidean Geometry organizes space by rigid figures and their (rigid) movements. Its key property is being "closed under homotheties" (its group of automorphisms contains the homotheties). By this, a theorem, a property of a figure, remains valid by enlarging or reducing at leisure its length, surface, volume ... . By this, the "local" or "medium sized" space of senses is perfectly unified with physical space, in the very large and in the very small. This property characterizes Euclidean Geometry w.r. to the non-Euclidean ones.

Note now that Euclid's postulates are "constructions": draw a straight line from any point to any point ... produce a circle with any center and a distance ... and so on so forth. "Theorem" means "vision", "scene", in Greek: by ruler and compass further constructions are "shown" by acting on space (first theorem, book I: construct an equilateral triangle on any straight line ... we all know how ${ }^{3}$ ).

21982 Field Medal, A. Connes works since the early '80's at the geometric foundations of Quantum Mechanics.

${ }^{3}$ The intersection point of the circles centered on the end points («the extremities of a line are points») is given by the intended "parmenidean" continuity of the (circular) lines, since «a point is that which has no parts» and «two breadthless length», i.e. two lines, produce, by intersection, "that which has no parts". That is, in Euclid, a point is given as the result of an intersection of two lines (this is observed by Wittgenstein as well). Only the formalist rewriting of Greek Geometry 
And this is all done by rule and compass only: these (ideal) "tools" make space mathematically accessible, they organize it, they allow to measure it, to operate on it. Nothing else is needed.

\subsection{B. Riemann}

Riemann's main aim is to account for the unexplained Newtonian "action at a distance". He tries to understand gravitation (but electromagnetism and heat propagation as well) by the "structure of space" ([Riemann, 1854]; see also [Boi, 1995], [Bottazzini\&Tazzioli, 1995]). This revolutionary approach may be partly found also in Gauss and Lobatchevski, but it reaches with Riemann its highest mathematical unity.

One of Riemann's concerns is to understand under which general conditions we may soundly measure. This is possible when rigid bodies are preserved, as by moving a rigid "meter" one may compare lengths. And here comes Riemann's general analysis of curbed spaces, which shows that spaces (manifolds) of constant curvature guaranty the invariance of measure (the rigidity of bodies). Euclidean spaces are a particular case of these manifolds, indeed the critical one, i.e. when the curvature is constantly 0 .

But the other spaces can make sense as well, since they can give an account, by geodesics, of these mysterious action at distance. Riemann dares to think that «the concept of rigid body and of $a$ light ray, non longer are valid in the infinitely small»: bodies may be no longer rigid, light may go along varying curbs .... The point is, and this is one of his major results, that the metric structure of a (riemannian) manifold, or its measure by a length, may bi-univocally related to the its curvature (the metric tensor and the tensor of curvature are related, in fully general situations). Moreover, it may make sense to analyze a space of non-constant curvature, as «the foundation of metric relations must be found elsewhere, in cohesive forces that act on it». A "divination", will recall $\mathrm{H}$. Weyl in the '20s, in reference to Relativity Theory: forces between bodies are related to the (local-metric) structure of space. And this approach «should be allowed if it would lead to a simpler explanation of the phenomena». Since Einstein's work, we understand the relevance of this extraordinary insight of Riemann's.

Thus the geometric organization of spaces may provide an understanding of physical phenomena, beginning with the analysis of measure and distance. For this purpose, Euclid's ruler and compass must be generalized, since $« \ldots$ in a continuous manifold the metric relations must be introduced on different grounds ${ }^{4} »$. Then, a linear element does not need to be represented as the square root of a second order differential form (Pithagoras' theorem), but more generally as $\mathrm{ds}^{2}=$ $\sum \mathrm{g}_{\mathrm{ij}} \mathrm{dx} \mathrm{i}_{\mathrm{i}} \mathrm{j}$.

This is how, for Riemann, we access, measure and operate on space, while understanding physical phenomena by Geometry. Then space manifolds are proposed, as a "genealogy of

could claim that this theorem is not soundly proved by Euclid, see [Heath, 1908] and one century long commentaries.

${ }^{4}$ Riemann's quotations, in brakets, are from [Riemann, 1854]. 
mathematical concepts", by making explicit some hypothesis, which ground the mathematical construction into phenomenal space: some key ones are, according to Riemann, connectivity, isotropy, continuity .... H. Weyl will add symmetries as one of the fundamental properties, which structure physical space.

Of course, by Riemann's distinction between the "local" and the "global" structure of space (the metric structure and the topological one, the latter related to the Cartesian dimension) a key aspect of the unity of Euclid's approach is lost: physical space, the space of microphysics ("the infinitely small") or of remote spaces, may have properties which escape the experience of senses. In Riemann's approach, the relation between local and global is the result of a complex and novel mathematics: the gluing of local maps by differential methods; homotheties do not allow any longer to transfer "medium sized" experience and knowledge to any scale. And this is extremely modern: from Relativity Theory and Quantum Physics, we learned that access, measure and operations, in the very large and the very small, cannot be provided by the naive analysis of senses.

Yet, there is a unity in Euclid's and Riemann's approaches, as stressed here. A synthesis is also given by [Poincaré, 1913] in a sentence: «faire de la géométrie, c'est étudier les propriétés de nos instruments, c'est a dire du corps rigide».

\subsection{A. Connes}

Given any topological and, thus, any metric space $\mathrm{X}$, one may consider the set of continuous functions, $\mathrm{C}(\mathrm{X})$, from $\mathrm{X}$ to the complex field, as a suitable algebraic structure (a commutative $\mathrm{C}^{*}$ algebra). $\mathrm{C}(\mathrm{X})$ is very important, as it includes the space of measures on $\mathrm{X}$.

A classic result of Gelfand allows to go the other way around. Given a commutative $\mathrm{C}^{*}$ algebra $\mathbf{C}$, it is possible to construct a topological space $\mathrm{X}$, such that $\mathrm{C}(\mathrm{X})=\mathbf{C}$. The points in $\mathrm{X}$ will be characterized by the maximal ideals of $\mathbf{C}$ and so on and so forth as for reconstructing the geometric structure of $\mathrm{X}$ on the grounds of the properties of $\mathbf{C}$.

In classical and relativistic physics measures happen to commute: the result of several measure operations does not depend on their order. This is not so in Quantum Mechanics. The measures of position and momentum of a particle, for example, do not commute. And this is crucial: in Quantum Physics these are the observables. Measure by instruments is the only access we have to "physical reality". More precisely, we can construct knowledge in microphysics only by setting up instruments for measure: there are no other observables. This is where we have to start. In this, there is a complete conceptual continuity w.r. to the approaches by Euclid and Riemann. But the "instruments" of measure do not have the relatively simple nature of the ruler and compass, even not in the generality of Riemann's notion of "rigid body" or of his "ds ${ }^{2}$. Measure is now given by the complex physical and conceptual instruments of microphysics: the only grounding certainty, which founds quantum mechanics, is given by a few observable phenomena, such as the noncommutativity of measure (and the related essential indeterminism).

Heisenberg first replaced classical mechanics, where observable quantities commute, by a "mechanics of matrices", where observable quantities do not necessarily commute. His algebra of 
matrices is then obtained from a groupoid, which replaces the classical (commutative) group of measures. Now, this latter group is the starting structure to reconstruct space, à la Gelfand: from a commutative algebra to a topological space. Connes' work, since many years, consists in reinventing Geometry from a non-commutative $\mathrm{C}^{*}$-algebra: beginning with measurable spaces, to topological, metric and differentiable ones ([Connes, 1994]). The differences are dramatic, as the very notion of point and of trajectory are different from the classic notions: there are no more maximal ideals (and trajectories are closer to the "paths" derived from Feynman's notion of integral). The debate is very lively (and difficult), but many agree that Connes' approach is gradually giving an account of the mysterious nature of some physical phenomena, at the level of microphysics, including non-locality (a particle is not "located in a point", in Quantum Mechanics). A crucial issue is the dependence of the reconstruction of space of microphysics on the order of measure: but this is how we access to it. Once more, Poincaré may be quoted for his insight. Even though it would be too much to attribute to him a "divination" concerning the possible Geometry of Quantum Physics, yet he observed: «Des êtres qui éprouveraient nos sensations normales dans un ordre anormal, créeraient une géométrie différente de la nôtre» [Poincaré, 1902].

\subsection{Some epistemological remarks on the Geometry of Physical Space}

Starting from what is accessible and grounds knowledge, the observables, Geometry proposes an organization of physical space, which makes phenomena intelligible. We have no other way to constitute knowledge, but starting from observable, measurable phenomena, even when this observability has nothing to do with our direct experience by senses. As we learned from Relativity and Quantum Physics, we may then need to give up the identity "space of senses = physical space", so beautifully proposed by Euclidean spaces and their closure by homotheties. Knowledge in very large and very small scales is constructed differently: no rigid ruler, no compass of "human size" may organize the spaces of galaxies and of elementary particles, by homotheties. Their intelligibility cannot be grounded directly in our senses, on our eyes, hands, by our movements and actions, normalized by Euclid's rigid tools, but must be mediated by complex instruments of observation and measure. These instruments are themselves the result of complex "theoretical commitments", as they are set up on the basis of an existing or proposed theory, or of strong hypothesis, beginning with the decision to measure "this and not that".

Yet, the only dramatic change, here, is related to cognition: the direct experience of senses is no longer sufficient to understand physical space, while there is unity in the method. It is surprising that we still have to digest this apparent cognitive discontinuity: the "ontological" commitment (Geometry is "space per se", beginning with Euclidean Geometry) did not allow to appreciate that the mathematical objectivity is in the construction, not in an ontology. There is no such a thing as "absolute space", but there is the objective reconstruction of a space of action, by the cognitive subject, with the contingent tools of active experience. Objectivity is reached when the cognizing ego is able to relativize his construction: fix one or more reference systems or "view points', and the forms of their communication/interaction; fix the tools for measure. Then the 
construction becomes objective. As long as the subject believes in absolute spaces (Newton), in "absolute laws of thought" (Frege), in "views from nowhere", there is no foundation for knowledge, but an artificially unified frame for illusory certainties.

In contrast to this, I stress that the method, from Euclid to Riemann and Connes is uniform and sound: access, measure and operate on space, with the appropriate and explicitly given tools, and organize it by one of our most beautiful conceptual construction, Mathematics, Geometry in particular. For an historical reference to this approach, note that Poincaré's critique of logicism and formalism proposes to supplement the foundational investigations in Mathematics «by a genetic analysis», the analysis of a conceptual genesis or construction [Heinzmann, 1998]. His understanding of Geometry as a genesis, beginning with the movements of rigid bodies, specifies Riemann's approach to Mathematics as a "genealogy of concepts" as well as Helmholtz's reference to "facts" (see [Nabonnand, 2001]); it is not an empiricist view nor rationalism, but a "phenomenological" understanding (cf. below and Husserl's fundamental text [Husserl,1933]). This neo-kantian understanding of Poincaré's views has been confirmed by many (see [Nabonnand, 2000] for references). Mathématics is not gounded on arbitrary conventions: these conventions are the most convenient choices («les plus comodes») for us, human beings, in this world, with our shared biological being. Poincaré's program, as we understand it, is a preliminary step to ground Mathematics in our reference to the regularities of the world that we see: we draw on the phenomenal veil on the grounds of our active, cognitive experience of it. The structures of Mathematics are conceptual proposals, meant to make this world intelligible ( $\ll \mathrm{Si}$ [la nature] offrait trop de résistance, nous chercherions dans notre arsenal une autre forme qui seerait pour elle plus acceptable», [Poincaré, 1898]). The role of action, proposing, understanding are crucial. The resistance of nature is deeply embedded in physicality and in our biological being, in the historical formation of sense. In a Manifesto on web [Longo et al., 1999], a modern version of what we would like to call "Poincaré's program" is defined as the "Cognitive Foundation" of Mathematics. The point, of course, is to go beyond introspection, the only tool these great mathematicians had (because also Riemann, Helmholtz, Enriques and Weyl shoud be quoted) and refer to modern Cognitive Sciences, as a scientific analysis of our practical action and conceptual reconstruction of the world (see also a conference held in Rome, September 2002, based on this program, a reference is in [Longo et al., 1999]).

Of course, the foundational program I am sketching here is an epistemological one: it is an analysis of "how" we access to knowledge, or of the "knowledge process". In Mathematics, spaces, objects and structures are constructed from the explicit assumption of cognitive grounds, and this is objective. This analysis has been programmatically disregarded by the logicist and formalist approaches to the foundation of Mathematics, in the XX century, as they only focused on (formal) proofs. This was a necessary investigation, but, unfortunately, it excluded the analysis of the constitution of concepts and structures and pretended to encode the world in formal strings of symbols. Now, there is no doubt that Mathematics is abstract and symbolic, but the one century long identification of these deep notions with "formal" excluded meaning and epistemology from 
the foundational analysis. We have to broaden the foundational project to the "constitutive path" of mathematical abstract structures, beginning by their meaningful grounding in (and their organizing) phenomenal space and time.

\section{Codings}

It is hard to appreciate how severe was the crisis of the 2300 years old Euclidean certitudes, in the XIX century, as induced by the non-Euclidean approaches. Frege's deep investigations started the modern "royal way out" from the novel problem of space. (Mathematical) Logic was explicitly contrapposed to foundational analyses grounded on phenomenal space. «The wildest visions of delirium ... remain so long as they remain intuitable, subject to the axioms of Geometry» ... absolute certainty can only be recovered with reference to the concept of number and the logical laws that govern it: «... the laws of arithmetic govern all that is numerable. This is the widest domain of all; for to it belongs no only the actual, not only the intuitable, but everything thinkable» [Frege, 1884, p. 20 and ff.]. Geometry itself (but Frege cautiously considers only Euclidean Geometry) can be found analytically on the notion of number, as relation between lengths [Frege, 1873, p. 9-10] (see the discussion in [Tappenden, 1995]).

In a different way, this program was fully developed by the subsequent work of Hilbert. His first and main foundational writing, [Hilbert, 1899], is a very relevant approach to the issue by formal tools. The foundational problem is reduced to the analysis of formal consistency: what only matters, in Mathematics, Geometry in particular, is the non-contradictory status of the axioms, with no reference to meaning, in space in particular. By a remarkable technical work, Hilbert gives all possible "relative consistency" proofs in Geometry: put an axiom, take another away (Euclidean, non-Euclidean, Desarguesian, non-Desarguesian, Archimedean, non-Archimedean ...) ... embed one system into the other. Beyond Beltrami-Klein's work, the relative interpretations of Lobatchevski's and Riemann's spaces in Euclid's are brought to the highest rigor and generality. Then a final masterpiece: formally encode, by analytic tools, Euclidean Geometry into Arithmetic. The following year, by posing, at the Paris conference, the problem of consistency of Arithmetic, the scientific program of formal foundation is fully given: no reference to meaning and space, nor to the way we access to knowledge of it; just prove formally that the axioms of Arithmetic do not entail " $0=1 "$. This is the foundational problem of Mathematics, including Geometry, of course, since the latter, by encoding, is just a subsystem of Arithmetic.

The extraordinary "tour de force" of Hilbert's is much appreciated by many, including Poincaré. In his review of Hilbert's 1899 book, he acknowledges the technical achievement, but he stresses as well the loss of meaning, the trivialization of our understanding of space, the senseless reference to Mathematics as codings of axioms into «le piano raisonneur de Stanley Jevons» from which «on verrait sortir toute la Géométrie». Elsewhere Poincaré, will refer to this view of Mathematics, which underlies the foundational programs of Peano, Padoa, Hilbert, as «la 
machine à saucisses de Chicago»: from pigs and axioms produce sausages and theorems (see [Bottazzini, 1999]).

As observed in [Girard, 2001], about half of the XX century may be considered the «time of codings». Hilbert's foundational program, centered on Arithmetic (the theoretical locus of codings), started the modern trend. These fantastic times, for Mathematical Logic, produced an amazing by-product: the coding of knowledge, viewed as deduction, in digital machines, beginning with Turing Machines. Turing's fundamental mathematical distinction between hardware and software, a distinction at the hearth of his Machine, is at the origin of modern computing. In particular, it started a "Theory of Programming", once programs (as software) have been mathematically differentiated from hardware. Moreover, the "Universal Turing Machine", which may encode any other Turing Machine and simulate it, gave us the notions of operating system and compiler. Poincaré could not imagine that the "sausage machine" was bound to go so far. This is how history goes: wrong foundational programs, based on provably wrong conjectures (formal decidability, completeness and finitistically provable consistency of Arithmetic), may have major fall-outs, when precise and robust. Also Laplace "analysis of (planetary) perturbations" was meant to give a complete account of the future (and past) of deterministic systems, governed by Newton's laws. Poincaré showed that it does not work (1890), but Laplace results and conjectures originated large part of the fantastic work in Analysis in the XIX century.

However, it is time to overcome wrong projects by reconsidering what is at the core of them. One key component of later developments of Frege-Hilbert ideas, roughly the foundational program of Mathematical Logic, broadly construed during the XX century, is the believe on the "transparency" of codings. More precisely, contentual information is preserved under any "reasonable" coding. One takes whatever fragment of Mathematics, encodes it into the axioms of Set Theory (or, better, Arithmetic, as numbers govern «everything thinkable»), proves the (relative) consistency of the intended system and the game is over (of course, one may subsequently feed by them a Turing Machine or a modern computer, under a suitable 0-1 coding.) This reduction is rarely done in practice, but it often had amazing consequences. Words do not suffice to praise the enormous amount of information we obtained from Set Theory and Proof Theory (I earned my life by applying the later, its constructive branch - Type Theory, to computing, see [Asperti\&Longo, 1991], [Longo, 2002]). And note that XXth century Proof Theory is the proof theory of Arithmetic, as, since [Hilbert, 1899], the key assumption (or aim) is that any structure, any deduction can be encoded into suitable extensions of Peano Arithmetic and then formally analysed, see footnote 5 . Of course, the positive impact of these views in founding digital machines have been enourmous, thus their large success. But also, (formal) Descriptive Set Theory, just to give a further example, unified scattered results in Mathematics, displayed the key underlying assumptions, proposed new relevant problems .... Not less than in Computer Science, the outcome has been immense. The logico-formal analysis remains a necessary component of the foundational work in Mathematics and Computing. 
Now we have to enrich this program by what is missing: sense and meaning, the reference to space, both as a cognitive matter and the locus for physical phenomena. In particular, we have to analyze knowledge by methods that are "sensitive to codings": so far this may be understood by the reference made in $\S .1$ to the structuring of phenomenal space, as an interface between us and physical reality, or the "veil" on which we draw Mathematics. But more will be said below5.

Let's conclude this section, by stressing that the very Proof Theory which originated this dominating paradigm (information and deduction do not depend on meaning nor on codings) is now being opened to radically new proposals. J.-Y. Girard, by Linear Logic first, and Locus Solum more recently [Girard, 2001] started an analysis of proofs "sensitive to codings" and where the artificial split of syntax and semantics makes no longer sense ${ }^{6}$. In these theories, the geometric structure of proofs is relevant to deductions: connectivity (recall Riemann) and symmetries (Weyl) govern the proof; that is, its geometric "diplay" is crucial. Of course, Riemann and Weyl referred to physical spaces, while here it is a matter of proofs: it is as if these properties of space, in Girard's systems, had "come back through the window" into Proof Theory, by structuring proofs. Note that, for Poincaré, premises must be related to conclusion by a "mathematical architecture"; moreover, in his fight against formalism, he hinted that mathematical reasoning is non-invariant w.r. to meaning [Poincaré, 1905, 1908] (see the discussion in [Heinzmann, 1998]). A remarkable insight into the incompleteness of formalisms (see also Weyl's conjecture of the incompleteness of Arithmetic in [Weyl, 1918] $)^{7}$.

\subsection{Geometry in computing}

Turing Machines have no space and yield a Newtonian time. As for space, theorems prove that one, two ... n-dimensional hardware (head and tape) does not modify their expressive power: up to

\footnotetext{
${ }^{5}$ Often historians stress that Hilbert was not a formalist. This is absolutely true: in several papers, even in the introduction to [Hilbert, 1899], in correspondence ... one can find Hilbert's major concern for structures and physical meaning, in Mathematics. Hilbert was an immense mathematician, not just the founder of modern Mathematical Logic. However, the technical perspective in the 1899 book, his Foundational Program, as specified from 1900 to the '20's, became the paradigm of formalism and have committed the century to an incomplete analysis of foundation, up to the recent revitalisation of Hilbert's Program. Along these more recent formalist guidelines, Euclid's and Riemann's, for sure, but probably even Connes' approach to physical spaces can be encoded in predicative subsystems of Second Order Arithmetic, [Simpson, 1999]. An informative analysis, as for relative consistency or consistency "strength", for example. But it entirely misses the relevance of Mathematics for knowledge of space and cognition. To this further aim, if one wants to refer to the remarkable debate at the beginning of the XX century, it is Poincaré's foundational program that must be revitalised today, not Hilbert's, as we pointed out.
}

${ }^{6}$ The very broad definition of "geometric" as "sensitive to codings" was proposed by J.-Y. Girard, in discussion, at a Workshop in Marseille, April 2001.

${ }^{7}$ The proofs of formally unprovable statements of Arithmetic use meaning along the proof (see [Longo,1999]); or, "geometric judgements" step in (well-ordering, as defined by formal induction over full second order comprehension principle is non effective, while it is simple - and "effective" - as a geometric judgement, see [Longo, 2002]. Symmetry is another geometric judgement, largely used in Mathematical Physics, in proofs). 
a linear time complexity encoding (or at most polynomial time, with a small exponent), the Cartesian dimension of the "physical" process does not affect the computation. Of course, this is so because these "machines" are a remarkable but purely logical construction: the reduction of the notion of sequential deduction to elementary steps (move right or left a head, write 0 or 1 on a tape). Physics is not there: space had been excluded from the foundational discussions since long. Have you ever seen a physical process, which does not depend on dimensions? In some cases, since Relativity, and even more so in modern string-theory for Quantum Physics, it is as if "only dimension matters".

As for time, in Turing Machines, it is not only absolute and linear, but it is actually generated by the clock. Now, in Physics, time is understood as a relational matter, once one goes beyond Newton's absolutes. Moreover, measuring time by the lonely clock of a Turing Machine is like having a meter in an empty Universe: there is no distance in that Universe, but just the meter.

In summary, Turing Machines are fantastic logic machines, they are not physical machine: they initiated us to the first steps towards a "logic of programming" and, thus, how to make machine work logically. Their main fall out has been a the invention of a Science of Programming, grounded on the fundamental distinction hardware and software. And the software, up to the recent challenges in concurrent programming (see below), has been designed for long on the basis of the main paradigms proposed by Turing and his contemporaries (Turing's approach gave us "imperative programming"; Church's $\lambda$-calculus originated "functional programming"; Herbrand's theorem, "logic programming"). The physics, beginning with the issues related to space and time, are out of the scope of these programming styles and, by this, they are turning out to be largely inadequate (or to require major "extensions") for the cuncurrent, asynchronous and distributed systems mentioned below.

We briefly discussed of the geometric intelligibility of space in the previous sections, but also physical time has been deeply analyzed during the XX century. The relativized, but reversible time of Relativity, the irreversible time scanned by bifurcations in Dynamical Systems (or in the Physics of thermodynamical or critical states), the even more complex time of Quantum Physics, all these proposed forms of time do not rely on an absolute and unique clock; they view time, to say the least, as a result of a "relation", or as the problem of synchronization of possibly asynchronous systems.

In the last few decades, it happened that machines, those very digital computers that where born from the head of Turing (and Peano and Hilbert), have been distributed in space, by engineers. These practitioners even dared to have them "concur" in the same computation. That is, possibly far apart processes are no longer individually isolated in a vacuum, but run in parallel, communicate and access at the same database. In the 60 's and early 70 's only parallelism was at stake, yet some pioneers understood the major scientific change, which is now heavily affecting computing.

Concurrency summarizes the new problems. The point is not the parallelism of computations, but that they communicate and share data and programs along the computation, from different 
locations in space. Moreover, there need not be a universal clock: processes may run with their own independent clocks.

First dramatic change: computations are no longer compositional. The entire Theory of Computability, born in the '30s, relies on compositionality: Herbrand-Gödel-Kleene recursive functions, Church's lambda-calculus (one of the author's main interests for long) and Turing Machines, of course, (all computationally equivalent) are obtained by "composing" a few base functions, terms or steps, respectively. Thus, their "mathematical semantics" is compositional or the analysis of the function computed can be done piecewise and then composed. In contrast to this, one cannot analyze a computation carried on by concurrent processes (or give its semantics or tell which function is computed) by analyzing each process individually and then "compose" the results, because processes interact along the computation. Even more so: they may compete in accessing the same database, which, once used by one of the processes, may change.

In order to appreciate the relevance of the latter problem only, consider a seat-reservation system, e.g. an airline reservation net of computers: in this distributed system, priority and synchronization of access to an ever-changing data base is crucial (while an agent is modifying the data base, the others should have no right to access to it: this is a typical inacessibility condition.) Suppose more generally that you have two processes, $x$ and $y$. In a sequential system, you may have " $x$ then $y$ " or " $y$ then $x "$, which mutually exclude each other and exclude any other possibility. Consider now the rectangle with side names $x$ and $y:$ the two sequential paths above are the composition along the borders and they go, with time, from the bottom-left vertice, $(0,0)$, to the top-right one, $(1,1)$, say. But, if the two processes interact during the computation and/or access to the same resource, a good representation of the possible computations is given by all (increasing) paths (functions), in the rectangle, which go from $(0,0)$ to $(1,1)$. The inaccessibility situations may be represented now as "holes" in the interior of the rectangle: when one process goes through a certain status or area, then the other cannot act (see the example above with seat reservations). One or more holes allow then to classify the paths by "homotopy classes": the same class contains paths that may be "continually deformed" one into the other (i.e. transformed reciprocally without crossing a hole).

And here the non-trivial mathematics of Homotopy Theory steps in. Spatio-temporal connectivity is the issue, which means homotopy or equivalence under some notion of deformation in n-dimensional manifolds (as many dimensions as there are processes). It is surprising to see early work by Serre, in pure Geometry, and non-trivial Algebraic Geometry being applied in this novel areas of computing (see [Goubault, 2000] for surveys and results).

But the situation differs from mainstream Geometry in a crucial point: irreversible time is everywhere present in these analyses. Of course, it cannot be a linear time, as already mentioned. Time is branching, like along the bifurcations of dynamical systems. A nice way to represent it, is given by suitably parametrizing the paths in the example above along time: irreversibility of time may then be given by assuming that the paths are increasing functions from (co-ordinate) $x$ to $y$ (as already hinted above, in parenthesis). This originated the notion of directed-path (or di-path) 
and some non-trivial work which deserved the name of di-homotopy (the homotopic analysis of dipaths).

Note now that, in concurrency, the nature of "feasible" vs. "unfeasible" changes, w.r.to classical (sequential) computability. Within the Turing frame, one could prove that the halting problem is undecidable, that there exists partial computations that cannot be extended to total ones etc. Now, different issues are at stake: is this "computation" (a path) accessible, as an element of a given homotopy class, in a certain n-dimensional manifold? Impossibility results, including time lower bounds, may then be given on the grounds of purely topological methods (various papers in [Goubault, 2000]).

The idea of coding all of this into Turing Machines makes of course no sense: concurrent systems do not deal with a finite string of 0 and 1 only, but have an input flow and an output flow. They are open to interaction with the environment. Not to mention the complex issue of relational and branching time which started this discussion. Simulation on sequential machines requires the construction of quotients on computation paths, but this "simulating modulo" is far from standardized or unique, it is often "ad hoc" or missing the proper issues and challenges of concurrency (see [Aceto et al., 2002]). For example, what really matters in these systems is "how" a computation evolves in space and time, more than the input-output relation: its ongoing spacetime structure is the "observable". In short, concurrent systems perform different tasks, whose understanding requires new questionning, a different insight (different observables).

Many open problems are posed. I can only mention the interest of "fault tolerant systems". Distributed systems clearly allow fault tolerance in a way inconceivable to sequential ones: (small) continuous deformations, within an homotopy classes, may represent fault tolerance. But precise mathematical characterizations are still missing. Synchronization as well may present further challenges. As a matter of fact, a system is "truly distributed" when time required to connect processes is about the order of magnitude of the elementary step of computation, within a process. Now, the latter is about one nanosecond, today. And light is so slow as to go only $30 \mathrm{~cm}$ in that time. Thus, a concurrent system, distributed over the surface of the hearth (different acceleration systems), may undergo relativistic problems, as for synchronization. Relativistic delays may be computed, but this is far from obvious. This problem does not seem yet to be taken enough into account, with few exceptions ${ }^{8}{ }^{-}$.

\footnotetext{
${ }^{8}$ This section (and this paper) is clearly not a survey, but it presents a viewpoint grounded on some specific results. Thus, there is no mention of many other approaches to concurrency, where space steps in in a different way. From Milner's CCS, for example, to the very recent "Spatial Logic" by Cardelli, the issue of space - under the form of communication, event structure ... - is not less crucial and breaks as well the "linear coding myth". Yet, those systems are to be viewed as very relevant "space sensitive" variant of the more classical analysis of computing as "deductive systems", which originated in lambda-calculus and Type Theory. Of course, these and others proof-theoretic approaches to Concurrency, are important tools for program specification and correcteness (see [Bahsoun et al., 1999], for example).
}

${ }^{9}$ See also [Aceto et al., 2002] for more on Concurrency. 
In conclusion, even digital computers, when finally embedded in physical space, hardly realise the functionalist project, according to which a sequential Turing Machine, once the world is encoded into it, may represent any physical system - including biological ones, of course. Distribution in space of these very machines is sufficient to change, well before the answers, the questions to be asked to the physical system, in order to understand it.

\section{Living in space and time}

In this section some remarks are made on Biology, as premises to a subject that will be just hinted: cognition. A key assumption is made here, about which one may well disagree: cognitive phenomena are a matter concerning life, from cell to man. Others may well be interested in cognition for non-material entities on Sirius, or for various sorts of computers, but these are different topics. The assumption here - but we may be wrong - is that brain is a material but living machine and, as for humans, it only works in its preferred ecosystem, the skull of a man living in History (in the broad sense of a communicating community, with a common memory). Of course, here and there, some cognitive performances can be isolated and transferred on machines, even on the clocks of the XVIIth and XVIIIth, the fantastic "statues d'automates" meant to implement all human functions. Yet, in our view, human cognition depends on life, even though it is not reducible to Biology, as a science, since it also depends on language and History. That is, our constructed, historical and ever evolving knowledge of life cannot, alone and as it is, provide a complete explanation of phenomena, which required, so far, different methods and tools of analysis, such as our sciences of human communication and History. A novel synthesis is required, and this is the actual challenge of modern Cognitive Sciences.

Let's though focus on life phenomena and on some mathematical challenges that are posed by them. These phenomena are first of all a spatio-temporal matter. Beginning with the three dimensional structure of DNA and the folding - unfolding of proteins (which are not "alive", but are the "bricks of life"), the dynamics of forms is at core of life processes.

The relevance of the spatial organization in biological descriptions should always be present to our minds, as it is the first step towards appreciating the complexity of structures whose functionality is entirely lost by any sort of "linear encoding", such as the description on the tape of a Turing Machine (see §. 2.1). And all relevant cognitive functions, we claim, are irreducible epiphenomena of life.

As a preliminary observation about complexity (and conceptual irreducibility) of cognitive and biological phenomena, recall that classical Computability Theory is "compositional" and that today's distributed and concurrent systems for computing (distributed in space) are no longer so, see $\S$. 2.1. And yet, by recursion, classical computing is already very expressive. More relevant non-compositional systems are the dynamical ones.

Analyze, for instance, the movement of two physical bodies, just governed by Newton's law of gravitation. Then consider two more, independently. Both two-bodies systems stabilize in 
orbits, as predicted by Kepler. If you put the four bodies together, by the famous analysis of Poincaré, a chaotic behavior, as an entirely new organization in space, emerges, and in no sound way the new "four bodies" geometric system can be considered the "composition" of the $2+2$ systems. Thus, it is sufficient to move from the one-dimensional tape of a Turing Machine, or any equivalent system of formal rules, and analyze distributed (concurrent) systems for computing or least gravitational systems, that entirely novel Mathematics is required. The functionalist myth of the "independence from codings and structures" of the cognitive phenomena, the most complex expression of life, breaks down when faced with the representation of least extensions to physical space even of a few digital computers or of a few gravitational bodies. If an artificial or natural phenomenon needs either of the two approaches above to be represented, in no way it can be reduced to or represented by a linear, compositional and space independent system, such as a Turing Machine. Of course, one can move higher and be content of encoding (or believe that it should be possible to encode) their mathematics (not the phenomena themselves!) into ... Peano Arithmetic, in the style of Hilbert's 1899 book. But this is a different analysis and, yet, enough theorems show the provable incompleteness of the formalist approach (see [Longo, 2002] for a recent discussion and references).

However, even though there surely is "concurrency" and "dynamicity" in life, we need a further step in conceptual complexity in order to grasp the kind of Mathematics eventually required for its representation, if at all possible.

All the systems above are essentially "one-scaled". A few laws at one "conceptual level" suffice to describe them: interaction of processes by digital signals, by gravitational forces ... and many other forms of possible "network structures", but all of one "type" or a few types, conceptually similar. And Mathematics is very effective for this (and, yet, we still need a good theory for concurrent computing, for the dynamics of true turbulence - Navier-Stokes equations describe satisfactorily flows only far from borders, where turbulence is at its high [Farge et al., 1996] - etc.).

Now, biological phenomena are essentially "multi-scale". Before discussing this concept, observe that an apparently multiscale Mathematics is that of fractals. Starting at one level of "magnitude" one may go to finer and finer insights into phenomena, at different scales. But the law is just one, indefinitely iterated. Sometimes living entities may develop in this way: there exist very effective descriptions of vascular and respiratory systems as fractals (see [Brown, 1999], [Nonnenmacher, 1994], [Bailly et al., 1991] for example). Maximizing exchange surfaces and irrigation volumes yields a mathematical law that beautifully applies. These are peculiar situations where life is only present by the growth factor and the analysis may be purely physical, as for the wax in a beehive.

For the purposes of this discussion, let's view living as an alternating hierarchy of at least two organization levels: autonomous biological individuals (cells), organized groups of them (organs), which in turn are integrated in a superior level and unity by their physiological function (and yield a new living unity). In [Bailly et al., 1993], it is observed that, in physico-mathematical terms, 
fractals geometries can be typically found in organs, while the interactions of biological units may be better associated to dynamical systems. That is, in some cases, Mathematics, by Fractal Theories, may give a good account of the relation between structure and function in organs, while, as for living units, this relation is better analyzed, whenever possible, in terms of dynamics.

Note, that even within cells, the smallest living entity, one may find organs: the external membrane and the cytoscheleton, first, but also some sort of internal membranes and "rails", microtubules, that play a key role in organizing cells' metabolism and reproduction. This is just the beginning of a view of complexity where the mathematical tools commonly used are already split into different theoretical frames, according to the "scale"; each claiming some descriptive completeness, but just for its level of investigation. Moreover, these two (already schematic) levels interact vertically and thus yield a novel, essentially multiscale system: when the scale changes, the Mathematics we use for its analysis changes as well.

\subsection{Multiscale phenomena and the mathematical complexity of the neural system}

When one considers brain and its functions, the most complex single object we happen to know, the situation is further enriched.

Neurons communicate: first, they exchange neurotransmitters of various chemical natures. Their functionality depends also on the shape of the post-synaptic receptor, which are complex proteins. The geometric shape of the latter (external shape and internal channels), determines the transfer of ions into the receiving neuron. Then a very rich biochemical cascade takes place. Proteins largely compose it and it plays a complex role, both in transmition and in facilitating/inhibiting the subsequent activation of receptors. Now, in proteins, as basic elements of life, the function is in their shape: these huge molecules interact in the metabolic/information exchange according to their three-dimensional folding.

Move then to a larger scale, that of a neuron as a whole. We should definitely consider neurons as six dimensional entities: three space dimensions, plus three more due to the shape of their response profile. It is too rough an approximation to treat neurons, mathematically, only as "thresholds elements". Of course there are thresholds and these are crucial, but they are as essential to communication between neurons as it may be the carrier wave in telecommunication. The fine geometric structure and the modulation of the activation profile of a neuron is also part of the neural way of elaborating information.

An important example is given by the neurons of the V1 visual cortex. Their response profile has the peculiar form of an extended, asymmetric gaussian, along a rectangle (or of an odd derivative of a gaussian, as one can observe some sort of "Mach bent" which accentuates contrasts). This rectangle gives the direction of the inspected border in the receptive field. In a sense, the V1 cortex gives the local orientation of a (virtual) border of an object in the visual field, or it makes a "derivative" along a curb. Then it "integrates" or "glues" all these local onedimensional maps (directions) by the complex connectivity of iso-oriented neurons (see [Petitot, 
2000], [Gilbert, 1992]). In both activities, the shape of the activation profile seems to have a very relevant role.

And now comes yet another scale or a further level of integration, that of neural nets, that many study by the Geometry of dynamical systems (see, for example [Hertz et al., 1991]). And, further on, assemblies of nets and assemblies of assemblies... Their complexity and the role of synchronization in their functionality are analyzed in [Edelman, 2000] (see also [Varela, 1999]).

The claim is that conscious cognitive functions appear at this latter level. However, all these "boxed" structures concur to the elaboration of information, which is largely a geometric matter: from the spatial folding of post-synaptic receptors and of proteins in the subsequent biochemical cascades within neurons, to the shape of the response profile, to the synchronization of networks, then assemblies, of neurons. And the interaction goes throughout all levels, horizontally and vertically: a psychological state may affect the functionality of some neurotransmitters, thus the lowest level, and ... vice versa (psycho-medicines act at the synaptic level).

This is a major challenge for Mathematics, if we will ever be able to invent suitable tools to give conceptual unity to the analysis of these multiscale systems, which seem inherent to life. The approaches based on isolating a single conceptual/mathematical level (the purely logical function, the finer analysis of dynamical forms of connectivity, the shapes of proteins...) are very important endeavors, but each is essentially incomplete, as a mathematical approach to cognitive and brain functions. And they are useful also in view of their fall-outs. As extensively said above, the logical analysis of the foundation of (mathematical) knowledge gave us fantastic digital machines, for the logico-formal manipulation of strings of symbols, but with a very "rigid" hardware and, originally, no space (nor "true" time). We may expect from the Mathematics of neural nets the next revolutionary machine, endowed with an evolving hardware, in space and time.

\section{Theories Vs Models}

There is a clear distinction, in Logic, between (formal) Theories and (semantic) Models. It is largely artificial, but it turned out to be very useful, so far. In Physics the distinction is not so sharp and it has a very different nature. Mathematical Models provide "local representations" of phenomena, by isolating one scale and a few properties in them; Theories, instead, are meant to have a "global" nature. Yet, of course, locality may be very large: Rutherford's atomic model is or yields a Theory. The point is that in both cases, Models and Theories, Mathematics is used as a tool for representing, organizing, correlating phenomena, by laws as general as possible. The description's generality and breath in question (as local vs. global) is a subtle matter as mathematical physics always aims to the highest generality: as soon as a single "fact" is observed, the physicist tends to transform it into a general law. Moreover, "facts", as already mentioned, are already the result of a theoretical commitment: set up these measure instruments, correlate this to that. And facts may be cut off from contexts: the very contours of physical objects are established by mathematical tools, on the interface between us and the world (the "phenomenal veil" of $\S .1$ and 
2). This gives them full generality. For example, there is no such a thing as a photon or a quark: they are the result of a theoretical construction, grounded on a few sparks, a trace on a screen, but leading to general principles. Their mathematical model is already a Theory. As much as Kepler's model of the planetary system is a Theory as well, in particular when explained in terms of Newton's principles (gravitation). One scale in Physics may suffice to comprehend a full universe; thus, Models border or intersect Theories.

It is not so in Biology. First, contours, membranes of cells, say, are already there, they are not drawn by us, by Mathematics, as in microphysics (but also planetary orbits are a conceptual proposal, a mathematical organization of the planets). Of course, in Biology, interpretations must be given, but the "ontology" is essentially different: the unity of a living entity forces itself throughout the phenomenal veil. Second, experiences and evidences are heavily context dependent: cutting off a living entity from its ecosystem may miss the very causal relations one is looking for. This gives the major differences, in general, between experiences in vivo and in vitro (in a neuron, the artificial fluid of an in vitro experience, its being cut off from three-dimensional connections etc. give lower firing rates, higher resistance, unreliable potentials ... [Jennings\&Aamodt, 2000])). The arbitrariness of the mathematical modeling, a further abstraction from the context, is even greater: the "intended" assumptions are out of control, as most are implicit. Soon or late the author will acknowledge that there are "hidden variables" not taken into account in the model; often, this is due to interactions with other scales, out of the scope of the given model. Thus, in contrast to Physics, Models in Biology are always poorer than phenomena. And all of this takes us far from "biological theoretizing". A Theory should propose general constitutive principles, which unify properties and "explain" them. Darwin's evolution is a Theory, Edelman's selective theory for the immune system is another. Some general principles are put into focus and have a broad explanatory nature, which fits all scales.

All these issues ("contours", context dependence, multiscale interactions) pose major challenges to Mathematics in Biology, as theoretical generality is its aim; in Physics, this is "more easily" obtained by Mathematics constitutive role in drawing physical objects and by the possible or discernible context independence of physical experiences, while both conditions essentially fail in Biology. Thus, the gap between the "local" nature of Models and the required "global" nature of Theories is much greater than in Physics, and Mathematical Biology seems to provide only models, so far.

Yet, even modeling, which is so important for iterating experiences, transferring knowledge ... conjecturing Theories, is so hard. Consider "latent potentials" in Evolution. There is, for example, strong paleontological evidence that the double jaw of some reptiles, living 200 millions years ago, originated the internal hear of birds and mammals ([Gould, 1982; 1989]). How can you model this? Which energy is minimized, if any, or which geodetics, in which mathematical space may simulate such a contingent evolution? There exist dynamic models of co-evolutive systems, as they are called, but, before discussing the problems they are faced with, let's consider another, related, feature of life. 
In Physics, we know how to deal with states close as well as far from equilibrium; but also critical states are well defined and treated. By definition, the latter are "temporary": a physical system doesn't stay long in a critical state (on the verge of a change of state). Yet, living entities, both biological units and species, permanently live in an "extended critical state", [Bailly, 1991]. Homeorhesis or Varela's autopoiesis are a theoretical appreciation of this fact; where homeorhesis means a dynamical reconstruction of an ever changing equilibrium, which is autopoietic when internally reconstructed. In short, we live as if we were running on a tight string; and we do it quite well. And so do species. There is no such a thing as "equilibrium" in phylogenesis or in ontogenesis: a non-artificial ecosystem is never in equilibrium, it is always evolving. Only death in a desert of stones is biological "equilibrium".

Many physicists work at co-evolutive dynamical systems in Biology and, by deep and powerful mathematical tools, they try to model features like the ones above. The problem of course is that there is no pre-designed space of phases where one could draw evolutionary geodesics: the phase space is co-constituted at the same time as the phenomenon to be described. They depend on each other, while interacting with billions of other phenomena, as unpredictable as the one above (the "latent potentials"). And this, along an extended critical state. Are there just "hidden variables", or missing parameters, to be discovered and inserted in the model? There seems to be more than this. Minor variations in the evolutive context, a mutation say, seem to create a new phase space: attractors which should describe the dynamics, not only need to be embedded in larger spaces (more variables), but seem to "swing" into different phase spaces. How to handle mathematically these changes, which may be "conceptual" changes? We are in a situation similar to the multiscale nature of biological phenomena, mentioned in $\S .3$ (and surely related to it), but with its own mathematical difficulties. Well before the proposal of algorithms, Mathematics and its applications grow by proposing novel conceptual frames, as pointed out throughout recent history by [Patras, 2001], possibly grounded in new forms of "access" to phenomena (in the sense of $\S$. 1) or to new objects of knowledge (Newton's revolutionary conceptual frame will be recalled below). And, as stressed in [Parrini, 1995], conceptual frames connot be reduced to nor analysed only in terms of linguistic symbols ${ }^{10}$.

The terminology used above (hidden variables) recalls Einstein interpretation of the EPR (Einstein-Podolsky-Rosen) paradox in Quantum Physics (see [Ghirardi, 1997]). For Einstein, the standard interpretation of non-locality and indeterminism was due to an "incompleteness" of the theory: some hidden variables had to be taken into account to yield a more "realistic" interpretation. Physicists (Bell, Aspect ...) were able to prove that it is not so: the theory is complete and non-

\footnotetext{
${ }^{10}$ Infinitesimal analysis, say, is not only a matter of "new symbols" or algorithms for solving equations. Moreover, the failure of founding actual infinity by formal Set Theories - whose formal consistency requires larger and larger infinite cardinals - confirms the limits of the purely linguistic approach; the foundation of the concept of infinity is in the genetic analysis of its "progressive conceptualization", see [Longo, 1999]. The same should be said as for Grothendieck's Toposes and Thom's geometric approach to scientific explanation (see [Patras, 2001] for more insightful reflections on these revolutionary aspects of XX century mathematics).
} 
locality, non separability, indetermism are essential. Or, that the difference in approach between Classical and Relativistic Physics on one side, and Quantum Physics on the other, is "epistemic" (it concern the roots and the tools for knowledge; some prefer to say that the difference is "ontological".) In this sense, also Physics is organised at different conceptual "scales", each requiring its specific mathematical tools (microphysics, dynamical systems and General Relativity, for example) and their unification is a major scientific challenge. Yet, each phenomenal level may be soundly analised in its autonomy, in contrast to the unavoidable unity of living beings, for which the "vertical" interactions of the many levels or scales is the key issue. Moreover, in Physics, some new mathematics is being constructed to give an account of the split and try to recompose it: in $\S .1$, we hinted to the different geometries used in Relativity and Quantum Mechanics and how they relate (note that we just tried to propose an epistemological unification, with no commitments to ontologies).

Biologists should try to give us a rigorous interpretation of the gap between (the use of Mathematics in) Physics and Biology, comparable to the one Quantum physicists proposed w. r. to more classical approaches, if this is so. The difference, they should tell us, is ontological (or epistemic), if any: here or there are the exact limits you encounter where treating these problems with tools from the Physics of dynamical systems or Quantum Mechanics (similarly as the Geometry of Relativity does not apply to microphysics). We need radically different tools .... Perhaps we could then try to invent more suitable Mathematics. Mathematics is an open conceptual construction and may be indefinitely enriched: fortunately, it is not God given, nor it is all already contained in and mechanically derivable from today's Zermelo-Fraenkel Set Theory or predicative fragments of Second Order Arithmetic. When Newton and Leibniz unified metaphysically distinct universes, the sub-lunar and the supra-lunar bodies and their movements, they did not use the Mathematics of projectiles well developed by the engineers of the time, largely based on Greek Geometry. They invented radically new concepts and tools, not contained in Euclid's notions and axioms, and dared to use the actual infinite to analyze finite movement (trajectories, speed, acceleration), a true revolution. Of course, there was a path through History, which lead to their ideas, but the dynamics of Mathematics swung by their work into a different conceptual space, which included infinitesimal analysis. And, by Gauss' and Riemann's Differential Geometry, this also changed Geometry. We need at least a comparable change of paradigms or conceptual enrichment of Mathematics in order to deal with biological phenomena: by their peculiar autonomy and contextual dependence, we cannot easily draw their mathematics on the phenomenal veil by "cutting them off" from their contexts and by giving them constructed contours. This, I believe, is the underlying methodological challenge for Mathematics in Biology, as Mathematics usually organizes the physical world, sets norms for it.

\section{Conclusion: epistemological and mathematical projects}


In the spirit of this lecture, I will now hint to some possible work directions coming out from the proposed perspective. The central theme, on one side, aims at (re-)embedding Mathematics and its foundation in phenomenal space and time, which Mathematics contributes to constitute. On the other, space and time may relate the very foundation of Mathematics, which has been isolated within the enclosed terms of its internal foundation (Hilbert's Metamathematics is a mathematical discipline), to other forms of knowledge, such as Physics and the Sciences of Life, whose phenomenalities are first of all a spatio-temporal matter.

\subsection{Epistemology}

The epistemological program has been spelled out in several places, in particular in $\S$. 1.4. The analysis of the "mode of access" to phenomenal space is a first step towards a "cognitive" foundation of Mathematics. Once more, this is not meant to replace the logic and formal analyses: these are "necessary but not sufficient" ([Weyl, 1927]). As they are necessary, they come first, but the XX century prevailing monomania of focusing only on the invariants of language and conscious reasoning (logic and formalisms), would be now a major limitation to further investigations, even in Computer Science (§. 2.1). Again, there is no doubt that there are logic and pure formalisms, in proofs, and that they even concern large part of them: it is the believe in their mathematical, or even "cognitive", completeness, that is wrong. Consider, say, Arithmetic or lambda-calculus, very close systems. A lot can be derived by purely formal tools: even consistency for the type-free version of the latter, as the Church-Rosser theorem is a beautiful and purely syntactic game (see [Barendregt, 1984]). But as soon as you get to Mathematics, which is typed, meaning and structures step in ${ }^{11}$.

Thus, we need to go further, in particular in the reconstruction of the knowledge processes that lead us to propose concepts and structures, beyond the sole analysis of proofs. Concepts and structures are constituted in the interface between us and the world, on that phenomenal veil over which we draw them in order to organize and make intelligible the world, by Mathematics. They originate on the regularities we "see", as living and historical being, and develop along History, in intersubjectivity and language. The objectivity of Mathematics is in this process.

Also the reflections proposed above, concerning the challenges for Mathematics in Biology, are not just meant as informal/technical considerations, but they are an attempt to analyze the peculiar interface by which life presents itself to us. The mathematical analysis of the difficulties should

\footnotetext{
${ }^{11}$ Normalization for typed lambda-calculi, as soon as they yield some expressivity, implies consistency of Arithmetic of various orders (see [Girard et al., 1989]), thus it implies wellordering properties of numbers or ordinals (of a "geometric" nature, see [Longo, 2002]). Yet, there are more purely formal non-obvious theorems. "Genericity" for second order lambdacalculus is an example, [Longo et al., 1993]. It is a type- or proof-theoretic "implication" that has no (semantic) model so far. On the other end, continuous "geometric" structures (Scott Domains) may step in the inductive load of a proof of purely combinatorial properties of recursive functionals (see [Longo\&Moggi, 1984], [Longo, 2001a]).
} 
stimulate a foundational investigation on the tools used and stress this constitutive role that Mathematics has w. r. to reality: these difficulties are due to the different "autonomy", criticity and multiscalar phenomenality of life, if compared to the physical one. In general, each analysis of the interface between us and phenomena, within different forms of knowledge or access to reality, bears a foundational character. To put in husserlian terms, Mathematics is a (key) component of the "phenomenal constitution", at the core of any analysis of knowledge.

Our focusing on the issue of space is not meant to present a new monomania, that of Geometry, but to enrich existing paradigms by what was programmatically excluded by the founding fathers, and for good reasons (at their time: we are no longer troubled, today, by Riemann's Geometry and, perhaps, even not by Connes'). Moreover, the Mathematics of space and time are "transversal" themes to different sciences. And the related foundational and methodological considerations should be an essential component of interdisciplinary researches. It is largely insufficient to transfer well-established algorithms from one discipline to another (physicists do so too often in relation to Biology). We have to be "monist of matter" not of the "method": different phenomenalities may need to be analyzed by different tools. Yet, an explicit reflection on the methodological differences and analogies may lead to a unification, which is never a matter of a transfer or superposition of techniques, but of a new invention, a new synthesis (recall the example mentioned of infinitesimal analysis; but the same could be said for the Geometry of manifolds or the non-commutative one, major steps forwards, which also unified previous approaches).

\subsection{Geometry in Information}

In §. 2, we focused on "codings". Hilbert's analytic encoding of all existing Geometries and Gödel's representation lemma to the Incompleteness Theorem (the metatheory is encoded in the theory, Arithmetic again) are "coding's" highest moments and marked the century. By the first, the foundation of Geometry was definitely considered as a subproblem of that of Arithmetic. The second started Computability Theory, by the invention of Recursive Functions and gödelnumberings. Turing added the encoding of the world into Machines, and of Machines into themselves. Foundation and knowledge were supposed to "pass through codings", or to be "coding independent". Shannon developed a Theory of Information on Turing's ideas: analyze information properties independently of its coding, as sequences of 0 and 1's or whatever. Thus, both Computability and Information Theory are coding insensitive (modulo minor complexity results). As said several times, this gave us immense and perfect digital database and network of communication. One can download the Encyclopedia Britannica and Mozart's concert for Flute and Orchestra from California in a few minutes. And the file may be copied as many times as required, exactly in its original form.

Of course, this has nothing to do with cognitive activities. Brain is slow. Our memory doesn't store the details and it is very bad at making copies; indeed, forgetting is its main feature, as a goaldirected oblivion is at the core of our procedural memory, of our constituting of invariants (including mathematical ones, [Longo, 2001]). Intersubjective communication is also slow, 
unreliable ... but very effective for its purposes, which radically differ from storing, copying and transmitting the digits of Encyclopediae or of Mozart's concerts. We remember and communicate meanings, forms, harmonies, emotions.... The claim, here, is that these processes depend also on the structure of their coding in our living brains.

We gave a very broad, very weak definition of "geometric" as "sensitive to codings", in conjunction to Girard's work (§. 2). This applies to the Geometry of space: code, for example, a finite dimensional Cartesian space $\left(\mathrm{R}^{\mathrm{n}}\right)$ into the real numbers $(\mathrm{R})$, by Cantor's method (the pair of real numbers $\left(0 . a_{1} a_{2} a_{3} \ldots, 0 . b_{1} b_{2} b_{3} \ldots\right)$ is associated to the real $\left.0 . a_{1} b_{1} a_{2} b_{2} a_{3} b_{3} \ldots\right)$. This is a bijection, easy to construct, but it misses even the weakest geometric property of space, as the notion of neighborhood is lost (the coding is everywhere discontinuous): that is, the topological structure is sensitive to Cantor's coding. Or, all the relevant informations concerning space (neighborhood, metric, ...) are lost. Technically, Cartesian dimension is a topological invariant and, thus, nothing is left after the set-theoretic "coding". But sensitivity to codings applies also to Girard's Proof Theory, a theory of spatial organisation of formulae along proofs, as well as it underlies the entire approach proposed here.

This issue is not just part of the continuum/discrete debate in the practice of Mathematics and in its foundation: it relates to it, but it is broader. Consider a discrete set of scattered points on a plane: a symmetry judgment about their structure in space is as relevant and autonomous as the inspection of the application of Modus Ponens, in a formal proof. The sequential encoding of the points and of all their spatial relations is unbearably complex and/or misses what matters, the symmetry. Indeed, physicists currently use judgments of symmetry in arguments and proofs; these suffice to deduce and convince as much as a logical rule. Symmetries pervade Nature, Arts, Mathematics, as beatifully synthetized in [Weyl, 1952]; recent neuro-physiological evidence stresses the deep physiological embedding of "symmetry judgements", as recognition of symmetric patterns ([Berthoz, 1997]).

As a long term project, it is time develop a "Geometry of Information", as an intrinsic mathematical theory (see http://www.di.ens.fr/users/longo for a preliminary proposal). What amount of information bears a "breaking of symmetries"? Does a change of shape yield a form of computation? Living neural systems can provide the starting ideas. In a sense, this approach is already present in the Theory of Neural Nets. Their dynamics is a form of elaboration of information, which is largely geometric (see [Amari\&Nagaoka, 2000], which mainly refer tough to the geometry of distributions of points, in the frame of a shannonian treatments of information). Thom's approach as well contains seminal hints in this direction ([Thom, 1972, 1990]). However, attention to phenomenal life is extraneous to Thom's Philosophy of Nature: a drop of wax or a jellyfish is mathematically the same and is molded by physical forces, when falling in water. It happens though that jellyfishes have morphogenes, which "do Geometry" by organizing growth, on the grounds of geometrically encoded information. As extensively discussed, this kind of models of living forms, are just models - though fantastic (see the fractal approach to vascular and respiratory systems in $\S .3$, but note that these are organs, not autonomous living entities). Thus, 
as models, they do not provide a Theory for biological phenomena, as in Thom's project, but at most one-scale models.

We should go further and look more closely at the structures of life. That is, on one side, we need to take into account those unique phenomena of life, such as function, reproduction and metabolism, which force a "contingent finalism" in every analysis. On the other, we need to refer to finer biological phenomena, w. r. to the Formal Neural Nets approach, beginning with the folding of proteins and the dynamic structure of dendrites (see [Percheron, 1987] for the latter: this structure seems to be "almost" fractal as their growth has some regularity of the sort, yet their morphogenesis is also due to neurotrophic factors, [Edelman, 1987] - a typical case of a blend of a physical and a goal-directed organization of living forms, whose analysis is an ongoing project). Both these scales, neurotransmitters and synaptic structures, contribute to the elaboration of information. Again, though, any one-scale analysis is far from providing a Theory.

Of course, the key feature of this Geometry of Information should be "coding sensitivity". It should be grounded on elementary regularities of space (symmetries, typically) and organize them in a non-compositional fashion. As suggested by Thom, the topological complexity of a structure or of a transformation could provide a quantitative measure, in a theoretical frame, which should mainly capture qualitative evolutions. Invariants and invariant preserving transformations should be analyzed on the grounds of the regularities one wants to preserve. Homotopy classes or mathematical grouping of "gestalts" could be given and preserved by suitable classes of continuous or differentiable or isometric maps.

The idea is that brain is a machine, which implements such a Geometry. But the Mathematics may depart from it, without any myth of providing a Theory of brain activities. Just a change in view point, possibly of method, w.r. to the 0 and 1 or thresholds' paradigms.

The difficulties of course are immense, also in view of the strength and depth of the Mathematics developed since Turing and Shannon, whose technological fall-outs have been changing our world.

\subsection{Geometric Forms and Meaning}

Let's conclude this programmatic paper by a dary claim on "meaning", such an indefinable notion. In reference to life, (changes of) forms are meaningful. Or, forms and their action/interaction in space contribute to "meaning". Consider a cell shaping itself or moving in space to preserve or improve its metabolism or while reproducing. For this cell an incoming signal or physical hit is meaningful. The signal's meaning is in the way it affects its goal-directed deformation or movement. And neurons, as cells, have a six dimensional form, in view of their response profile, an electrostatic matter (§.3.1). Thus, a signal, including an electric one, is "meaningful", per se, for a neuron, according to the way it participates to its ongoing activity or metabolism by the "deformation" it induces.

Then, meaning affects networks of neurons, assemblies of nets etc., by their spatio-temporal shapes as well, and its variations. Note though that the constitution of meaning happens in a non- 
compositional fashion, as defined in $\S .3$, and in no way the contextual meaning of a human linguistic expression, say, rich of intersubjectivity and History could be reduced to synaptic spikes nor to the geometric activity of a neural net. Meaning for complex living entities is in the relation between a neural activity (as "evolving form" of a network) and its context of life. No reconstruction of meaning is possible by reading just a neural activation or deformation: at the same time one has to consider the "ecosystem" and, for humans, the intersubjective and historical experience of it. Meaning is a relational/ interactive matter, where one of the components of the relation includes living entities and their forms.

The claim then is that all living forms and their variations are carriers of meaning, of its coconstitution, by the interplay between the evolving form and its context. Or, this is where meaning originates or it is rooted.

Later comes the organization of meanings at several scales, up to the richness of our communicating human community. The scientific challenge, in Cognitive Sciences, consists in being able to go up and down, from one scale to another, without necessarily assuming a reductionist approach, but by comparing and establishing interactions of different methods, which face different phenomenalities and different levels of meaning, from cell to History. Novel syntheses are a further task, never obtained, in the past, by pure transfer of techniques.

Of course, once the artificial split between formalisms for deducing, on one side, and semantics, on the other, was proposed, we could construct fantastic formal-computing machines and their programming languages. But then a dramatic question popped out: where is meaning? How comes that strings of binary digits may carry meaning? This is a problem, of course, for programming languages, machines and for coding independent Information Theories: strings of 0's and 1's or formal languages need to be decoded and interpreted (compiled). Living beings, instead, when elaborating or transmitting meanings, harmonies, emotions ... induce deformations in living neural systems which carry these contents in their geometric encoding and its variations. Thus, the cognitive challenge, if one associates meaning and information to living forms and to their evolving geometries, is in the understanding of the non-compositional co-constituting of sense, from elementary living entities up to our historical beings, as nested interaction of phenomena. The multiscale nature of this process is one of the major mathematical challenges.

Acknowledgements In the last few years, the activity in two working groups (CeSEF, on the epistemology of Quantum Physics) and 'Géométrie et Cognition', has been a fantastic occasion to meet and discuss with several colleagues in Physics, Biology and Philosophy. I am particularly indebted to the joint work and uncountably many discussions with Francis Bailly, Jean Petitot, Bernard Teissier, Catherine Vidal, Paul Bourgine, Rossana Tazzioli, Mioara Mugur-Schachter.

\section{References}

(Preliminary or revised versions of Longo's papers are downloadable from http://www.di.ens.fr/users/longo ). 
Aceto L., Bjorn V., Longo G. (eds.) The difference between Turing Computability and Concurrent Systems Special issue, Mathematical Structures in Computer Science, Cambridge U.P., to appear, 2002.

Amari S., Nagaoka K., Methods of Information Geometry, AMS Translations of Mathematics Monographs, vol 191 (translated by D. Harada), American Mathematical Society (AMS) and Oxford University Press, 2000.

Asperti A., Longo G. Categories, Types and Structures, M.I.T. Press, 1991.

Bahsoun J., Fiadero J. and Galmiche D. (eds) Proof Theory of Concurrent ObjectOriented Programming, Special issue, Mathematical Structures in Computer Science, Cambridge U.P., vol. 9, n. 3, 1999.

Bailly F. L'anneau des disciplines, numéro spécial de la Revue Internationale de systémique, vol. 5, n. 3, 1991.

Bailly F., Gaill F., Mosseri R. "Orgons and Biolons in Theoretical Biology", Acta Biotheoretica 41, 3-11, 1993.

Bailly F., Gaill F., Mosseri R. "Morphogenèse et croissance biologique : un modele dynamique simple pour le poumon" dans La biologie théorique a Solignac, Edition Polytechnica, 65-94, 1994.

Barendregt, H. The lambda calculus: its syntax and semantics, Revised edition, North Holland, 1984.

Berthoz A. Le sens du mouvement, Od. Jacob (english transl., Harvard U.P.; trad. italiana presso Boringhieri), 1997. (Review downloadable from http://www.dmi.ens.fr/users/longo.)

Bottazzini U., Poincaré, Le Scienze, 1999.

Bottazzini U., Tazzioli R., "Naturphilosophie and its role in Riemann's Mathematics", Revue d'Histoire des Mathématiques n. 1, 3-38, 1995.

Boi L. Le problème mathématique de l'espace, Springer, 1995.

Brown J.H., West G. Scaling in Biology, Santa Fe Institute Publication, 1999.

Connes A. Non-commutative Geometry, Academic Press, 1994.

Edelman G. Neural Darwinism, Basic Books, 1987.

Edelman G. A Universe of Consciousness. How Matter Becomes Immagination, Basic Books, 2000.

Farge M., Kevlhand N., Perrier V., Goirand E. "Wavelets adn Turbulence", Proceedings of the IEEEE, 84, n. 4, p. 639 - 669, 1996

Frege G., "On a Geometrical Representation of Imaginary Forms in the Plane", in Collecteed Papers on Mathematics, Logic and Philosophy, McGuiness, B. (ed.), Basil Blackwell, Oxford (1984), 1873.

Frege G. The Foundations of Arithmetic, 1884 (english transl. Evanston, 1980).

Ghirardi G.-C. Un'occhiata alle Carte di Dio, Il Saggiatore, 1997. 
Gilbert H. "Horizontal integration and cortical dynamics", Neruron, 9, p. 1-13, 1992

Girard J.Y., Lafont Y., Taylor R. Proofs and Types, Cambridge University Press, 1989.

Girard J.-Y. Locus Solum, Special issue, Mathematical Structures in Computer Science, Cambridge U.P., vol.11, n.3, 2001.

Goubault E. (ed.) Geometry in Concurrency, Special issue, Mathematical Structures in Computer Science, Cambridge U.P., vol.10, n.4, 2000.

Gould S. J. The Panda's Thumb, WW. Norton, 1982.

Gould S. J. Wonderful Life, WW. Norton, 1989.

Heath T.L. The Thirteen Books of Euclid's Elements, Cambridge Univ. Press, 1908.

Heinzmann G. "Poincaré on understanding Mathematics", Philosophia Scientiae (143-160), 1998.

Hertz J., Krogh A. and Palmer R. Introduction to the Theory of Neural Computation, 1991.

Hilbert, Les fondements de la géométrie, 1899 (trad. fran., Dunod, 1971).

Husserl E., The origin of Geometry, 1933.

Jennings C., Aamodt S. Computational Approaches to Brain Functions, Nature Neurosciences, Suppl., vol. 3, November 2000.

Longo G., Moggi E. "The Hereditary Partial Recursive Functionals and recursion theory in higher types" Journal of Symbolic Logic, 49 (4), 1319 - 1332, 1984.

Longo G., Milsted K. and Soloviev S., "The genericity theorem and the notion of parametricity in the polymorphic Lambda-calculus" Theor. Comp. Sci. vol. 121, 1993.

Longo G., Petitot J., Teissier B. Géométrie et Cognition, a manifesto and a research project, available on http://www.di.ens.fr/users/longo , 1999.

Longo G. "Mathematical Intelligence, Infinity and Machines: beyond the Gödelitis" Journal of Consciousness Studies, special issue on Cognition, vol. 6, 11-12, 1999.

Longo G. "Memory and Objectivity in Mathematics", Le Réel en mathématiques, à paraître, 2001.

Longo G. "Some Topologies for Computations" invited lecture, colloque Géométrie au XXème siècle: 1930 - 2000, Paris, September 2001a (to appear).

Longo G. "On the proofs of some formally unprovable propositions and Prototype Proofs in Type Theory" Invited Lecture, Types 2000, Durham (GB), Dec. 2000; Lecture Notes in Computer Science, vol. 2277, Springer, 2002.

Nabonnand P. "Les recherches sur l'oeuvre de Poincaré", Gazette des Mathématiciens, n. 85, Juillet 2000.

Nabonnand P. "La génèse psycho-physiologique de la géométrie selon Poincaré", à paraître dans La revue d'Histoire des Mathématiques, 2001. 
Nonnenmacher T.F., Losa G.A., Weibel E.R. Fractals in Biology and Medicine, Birkhauser, 1994.

Parrini P. Conoscenza e Realta', Laterza, 1995.

Patras F. La pensée mathématique contemporaine, Press Univ. de France, 2001.

Percheron G., "Principles and Methods of the graph-theoretical analysis of binary arborescences, J. Theor. Biology, 99, p. $509-552,1987$

Petitot J., Varela F., Pachoud B. Roy J.-M. Naturalizing Phenomenology: issues in comtemporary Phenomenology and Cognitive Sciences, (J. Petitot et al., eds) Stanford U.P., 1999.

Petitot J. Complexité neurodynamique en sciences cognitives, Rapport CREA, Ecole Polytechnique, 2000.

Poincaré H. La Science et l'Hypothèse, Flammarion, Paris, 1902.

Poincaré H. La valeur de la Science, Flammarion, Paris, 1905.

Poincaré H. Science et Méthode, Flammarion, Paris, 1908.

Poincaré H. Dernières Pensées, Flamarion, Paris, 1913.

Riemann B. "On the hypothesis which lie at the basis of Geometry", 1854 (english transl. by W. Clifford, Nature, 1873; trad. italiana e commenti di R. Pettoello, Boringhieri, 1999).

Simpson, S. Subsystems of second order arithmetic, Springer, 1999.

Tappenden J. "Geometry and generality in Frege's philosophy of Arithmetic" Synthese, n. 3, vol. 102, March 1995.

Tazzioli R. "An History of the Geometric Foundations of Mathematics: late XIX century" Revue de Synthèse, à paraître, 2002.

Thom R. Stabilité structurelle et Morphogénèse, Benjamin, Paris, 1972.

Thom R. Apologie du Logos, Hachette, 1990.

Varela F. "The specious Present: A Neurophenomenology of Time Consciousness" in [Petitot et al., 1999].

Weyl H. Das Kontinuum, 1918.

Weyl H. "Comments on Hilbert's second lecture on the foundations of Mathematics." [1927] in van Heijenoort J., From Frege to Goedel, 1967.

Weyl H. Philosophy of Mathematics and of Natural Sciences, 1927 (english transl., Princeton University Press, Princeton, New Jersey, 1949).

Weyl H. Symmetry, Princeton University Press, 1952. 